\title{
CONSIDERAZIONI SU ALCUNI SIGILLI REALI ITTITI*
}

MIRJO SALVINI

CNR. Roma

IL «SIGILLO DI MALNIGAL» (SBo I 84 = HGB I 159)

Th. Beran fornisce una nuova copia (fig. 1 b) e una lettura corretta di SBo I 84 (fig. 1 a): 'Mur-ši-li" SALTa-[wa-na-an-n]a? $\times 1 \times$ SAL.LUGAL '. La importante novità rispetto alle letture precedenti del Forrer ${ }^{2}$ e del Güterbock ${ }^{3}$ consiste nell'aver riconosciuto il nome Ta[wanann]a, identificata con quello di Tawananna III, la regina che era stata in carica nell'ultima fase di regno del padre Suppiluliuma.

Nel commentario a questo sigillo, per più versi anomalo, egli osserva come sia difficile ricostruire quanto si trovava fra il nome proprio Tawananna e il titolo SAL.LUGAL, ma che le tracce che precedono immediatamente SAL.LUGAL potrebbero corrispondere al segno LUGAL. Avremmo così i titoli di ambedue i titolari del sigillo così come i loro nomi, l'uno dopo l'altro senza connessione, e

Abbreviazioni:

2 BoTU = E. Forrer, Die Boghazköi-Texte in Umschrift, Zweiter Band, Leipzig 1926.

HGB I = Th. BERAN, Die hethitische Glyptik von Boğazköy, I Teil (BogazköyHattusa V), Berlin 1967.

$\mathrm{HH}=\mathrm{E}$. LAROCHE, Les hiéroglyphiques hittites, Paris 1960.

L. seguito da un numero, si riferisce al segno luvio geroglifico del manuale di E. Laroche, HH.

SBo I = H. G. GUTERBOcK, Siegel aus Boğazköy, Erster Teil (AfO Bh. 5), Graz 1940.

1 HGB I, p. 33 e 69.

2 E. FORRER, 2 BoTU, p. 14*. Va notato che egli vi aveva giustamente riconosciuto un sigillo di Mursili, mentre il Güterbock l'avrebbe poi attribuito a Urhi-Teššub.

${ }^{3}$ SBo I, p. 17 sg. 
privi del titolo GAL «grande». Quanto precede LUGAL rimarrebbe comunque oscuro.

Basandomi sulla copia di Beran, e in attesa di una collazione sull'originale, io credo che quelle che sono evidentemente le tracce residue della parte inferiore di due distinti segni cuneiformi, possano dar adito alla lettura LUGAL.GAL. La traccia che il Beran vorrebbe leggere LUGAL è infatti perfettamente compatibile con il segno GAL, di cui resta solo il cuneo orizzontale inferiore, mentre quanto precede, anche se imperfettamente copiato, mostra una complessità che ben può attagliarsi al segno LUGAL.

Propongo pertanto la lettura LUGAL.GAL. In tal modo viene superata la più grande difficoltà incontrata del Güterbock ${ }^{4}$, il quale proprio a causa della supposta mancanza del titolo LUGAL.GAL riteneva che il nome Mursili facesse parte della genealogia, per cui supponeva l'esistenza di un cerchio esterno dove ricostruiva nome e inizio della genealogia di Urb̧i-Tešsup. Questa integrazione conferma ad ogni modo l'attribuzione, che è del Beran, di questa impronta al sigillo di Mursili e Tawananna III, vale a dire la terza regina che assume questo titolo come nome personale. Avremmo così la successione asintetica dei titoli e dei nomi del re e della regina in carica. Manca comunque all'inizio l'abituale NA ${ }_{4}$.KIŠIB.

Nell'iscrizione della corona circolare, dopo SAL.LUGAL è omesso il prevedibile GAL, probabilmente per ragioni di spazio; abbiamo qui un'abbreviazione come quelle riscontrate nel sigillo RS $17.380+382 \mathrm{di}$ Mursili II s, in URU Ha-at<-ti>, e nel sigillo di Ašmunikal, del Museo del Louvre ${ }^{6}$, nel nome di Ni-kal-ma<-ti>.

L'iscrizione del cerchio esterno, ovvero corona circolare, doveva essere pertanto la seguente:

${ }^{\mathrm{m}} M u r-s i-l i$ AL $T a-[w a-n a-a n-n] a$ [LU]GAL.G[AL] [S]AL.LUGAL<.GAL> Mursili (e) Taw[anann]a - G[ran r]e (e) <Grande> [re]gina.

Per quanto riguarda l'iscrizione nel cerchio, non abbiamo problemi di lettura, ma di interpretazione. Una iscrizione cuneiforme vi è disposta nel modo seguente:

\footnotetext{
${ }^{4}$ SBo I, p. 46 sg.

${ }^{5}$ Ugaritica III (1956) fig. 7-8 e tav. II (Schaeffer) e p. 104 sgg. (Laroche).

${ }^{6} \mathrm{~V}$. il mio contributo in stampa in Syria 1990: “Un sceau original de la reine hittite Ašmunikal».
} 
ma-al-ni

GAL

SAL.LUGAL

Al Güterbock risale l'interpretazione, seppure espressa in forma dubitativa, che qui sia da riconoscere un nome proprio, diverso da quello della corona circolare (che lui, seguendo il Forrer, riteneva fosse $\mathrm{Ta}[\mathrm{nu}] \mathrm{be}[\mathrm{pa}])$, accompagnato dal titolo di regina: Ma-al-ni-gal SAL.LUGAL, oppure Ma-al-ni SAL.LUGAL.GAL. Non vi erano e non vi sono prove cogenti in favore dell'una o dell'altra lettura. Il Güterbock' optava per Ma-al-ni-gal ed accostava a questo nome cuneiforme il nome geroglifico di una regina nella serie SBo I 30-36, scritto $\mathrm{x}-n u / a-\mathrm{x}-l a$, che si è riscontrato in seguito anche nella bella

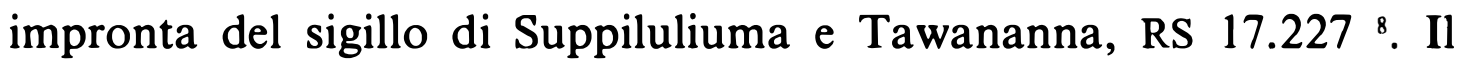
Laroche in un primo tempo ${ }^{9}$ non rifiuta totalmente l'equazione $\mathrm{e}$ poi ${ }^{10}$ sembra accettarla senz'altro.

Il Beran "accetta la lettura X-na-y-la, e ascrive i sigilli HGB I 213a-219 a Mursili II e Tawananna III, sua madre. Egli mette in dubbio, nonostante la concomitanza in HGB I 213a // 214a (fig. 1 c-d) di Mursili e Tawananna e della grafia suddetta L.271-na-L.292la ${ }^{12}$, che Malnigal sia veramente l'altro nome di Tawananna, e conclude che "eine sichere Lesung des Namens ist also vorerst nicht möglich». Questo è in sintesi lo stato della questione. In sostanza la sola possibilità che vi sia una corrispondenza con la grafia geroglifica $\mathrm{x}-n a-\mathrm{y}-l a$, è che il nome sia effettivamente Malnigal e non solo Malni.

Aggiungo qui alcune considerazioni sul testo del campo centrale. Tale iscrizione, che si inserisce in un cerchio sproporzionatamente grande, costituisce di per sé un'eccezione, poiché non si conoscono altri esempi di sigilli reali che rechino in questa posizione il nome proprio del proprietario del sigillo, espresso in cuneiforme invece che in geroglifico. La doppia legenda cuneiforme, nella corona

${ }^{7}$ SBo I p. 18 c. n. 52 e p. 47.

${ }^{8}$ Ugaritica III (1956) figg. 1-4 e Tav. I (Schaeffer), pp. 98 sgg. (Laroche).

9 Ugaritica III (1956) p. 100, e HH p. 286, indice s.v. Malnigal?, con riferimento ai due segni ancora illeggibili L. 271 e 292, per cui la scritta sarebbe 271-na-292-la).

10 Les noms des Hittites, Paris 1960, p. 111.

$"$ HGB I, p. 40 sub n² 213a = SBo I 30A, e pp. 74-75.

12 La situazione si ripete anche nelle impronte di Ras Shamra, v. E. LAROCHE, Ugaritica III (1956) p. 99. 
circolare e nel cerchio, è di per sé tipica della categoria dei «TabarnaSiegel" ${ }^{13}$; ma questi recano al centro uno o due segni cuneiformi che esprimono buon augurio (TI SIGs) (fig. 1 e) oppure uno o due segni geroglifici che hanno la stessa funzione (fig. $1 \mathrm{f}$ ), mai un nome di persona. In un caso abbiamo il segno geroglifico per "scriba" (SBo I $91=$ HGB I 148).

Esprimo l'ipotesi che l'indubbia anomalia di questo documento sia dovuta ad una "contaminatio" tra una concezione grafica e compositiva geroglifica e una esecuzione cuneiforme. La ragione di questo sarebbe da ricercare nella personalità dello scriba. Tento di spiegare in questo modo la notata mancanza di un nesso sintattico "cuneiforme" nella legenda della corona circolare, la cui simmetria fra nomi e titoli ricorda le soluzioni geroglifiche dei sigilli a edicola. Quanto alla legenda del cerchio si è notata la strana posizione del GAL sopra il LUGAL: se si opta per la lettura Ma-al-ni (o ma-al-ni) SAL.LUGAL.GAL, potrebbe appunto trattarsi di un influsso geometrico-spaziale del geroglifico, che riprodurrebbe la posizione della voluta (L. 363, MAGNUS), al di sopra del segno L. 17, REX, o al segno L. 15, DOMINA, per cui L. 363 sormontato da L. 15 vale DOMINA + MAGNA cioè "regina». Il GAL potrebbe tutt'al più valere per ambedue, nome e titolo.

La legenda del cerchio si può dunque teoricamente leggere $\mathrm{Ma}$ al-ni-gal SAL.LUGAL<.GAL〉, "Malnigal, 〈Grande> Regina», oppure Ma-al-ni SAL.LUGAL.GAL, "Malni, Grande regina», né la mancanza del determinativo SAL è di per sé elemento sufficiente a negare a quella successione il valore di un nome di persona, dato che abbiamo dei confronti nei «Tabarna-Siegel» di Alluwamna e di Huzziya (HGB I, 146a e 147), dove i nomi in cuneiforme sono privi del determinativo di persona.

Va però ricordato nuovamente che nessuno dei due nomi possibili, *Malni o *Malnigal, è attestato in altre fonti, e, allo stato attuale di conoscenza del luvio geroglifico, in attesa di una lettura più sicura del nome geroglifico 271-na-292-la, non vedo alcuna necessità di mantenere l'equazione proposta.

È stato osservato dal Güterbock che Malnigal è un ulteriore nome femminile composto mediante l'elemento -nikkal (Ningal),

${ }^{13} \grave{\text { È la }}$ «Gruppe XIII» del Beran, HGB I p. 66 sgg. Ma il sigillo di cui qui si discute (ne 159) viene inserito dal Beran all'inizio del XIV gruppo, che potrebbe quasi prender nome, come egli spiega, di "Tawananna-Siegel». 
come Nikal-mati e Ašmu-nikal, ed in ragione di ciò il Laroche lo registra come Malnikal fra i nomi delle regine hurrite ${ }^{14}$. A questo si deve però opporre che * mal non è un elemento hurrico, e che d'altra parte sembra difficile immaginare che una principessa babilonese cassita, com'era l'ultima moglie di Suppiluliuma, portasse un nome hurritizzante.

In ragione di tutti gli elementi vagliati, non resta che interpretare $m a-a l-n i$ in modo diverso, come un termine ancora sconosciuto del lessico ittito-luvio, che abbia una funzione augurale come gli esempi conosciuti dei cosiddetti «Tabarna-Siegel».

Faccio anche riferimento alla formula augurale TI SAL.LUGAL, che nel sigillo Mşt 75.10 di Maşat si accompagna, nel cerchio, ai nomi geroglifici del re e della regina (v. più avanti, e fig. 11 ). Penso quindi che sia il caso di esplorare la possibilità di un'equazione funzionale $e$, in seconda istanza, lessicale fra il sumerogramma TI "vita" (con o senza SIGs "salute») (fig. 1 e) che è particolarmente presente in sigilli di Mursili (cf. HGB I 174-179), il simbolo della rosetta (L. 189), spesso accompagnato dal geroglifico L. 369 (VITA) e dal triangolo L. 370 che significa qualcosa come «benessere, salute» (fig. $1 \mathrm{f}$ ), e il termine $m a-a l-n i$ del sigillo qui preso in considerazione. La scritta interna al cerchio di HGB I 159 potrebbe dunque significare "vita (o salute) alla grande regina».

L'impronta dovrebbe pertanto venir considerata come appartenente al sigillo della regina Tawananna III, che era in funzione durante il primo periodo di regno di Mursili II, il cui nome ricorre nella iscrizione della corona circolare.

SBo I $36=$ HGB I 219 (fig. 1 g, h)

Il Beran ascrive dunque i sigilli HGB I 213-219 a Mursili + Tawananna. Ma mentre per HGB I 213-218 (=SBo I 30-35) questo è sicuro, come già riconosceva il Laroche ${ }^{15}$, credo che per SBo I $36=$ HGB I 219 l'attribuzione debba essere un'altra, vale a dire a Suppiluliuma. In SBo I 36 (fig. 1 g) il Güterbock leggeva i resti cuneiformi $[\ldots] \mathrm{x}-r a \quad \grave{u} \mathrm{x}[\ldots]$, e la presenza del segno $\grave{u}$ gli rendeva difficile

${ }^{14}$ Les noms des Hittites, p. 357.

15 Ugaritica III (1956) p. 99, e aggiunge: «on ne peut décider le cas du $n^{2} 36$, où le nom du roi est perdu". 
l'interpretazione, che tendeva comunque a restituire KÁ.DIN]GIR.RA. Egli ascriveva tutta la serie di impronte ad Urbi-Teššup, ipotizzando che anche costui avesse preso una sposa da Babilonia.

Il Beran dal canto suo legge e interpreta HGB I 219 (fig. $1 \mathrm{~h}$ ):

[NA4.KIŠIB SALTa-wa-na-an-na SAL.LUGAL.GAL DUMU.SAL LUGAL KUR KÁ.DINGI]R.RA ̀̀ [.....].

[Siegel der Tawananna, der Grosskönigin, Tochter des Königs von Babyl]on und [.....].

Io propongo invece la lettura seguente: ...KÁ.DING]IR.RA ${ }^{\mathrm{KI}}$ $\mathrm{N}\left[\mathrm{A}_{4} . \mathrm{KIŠIB} . .\right.$. In effetti il segno che veniva letto $\grave{u}$, può benissimo leggersi KI se si prescinde, come da accidentale scalfittura, dal piccolo cuneo orizzontale che, nelle copie, segue il cuneo triangolare. Già le copie di Güterbock e di Beran divergono un po' al riguardo, e quella più recente, collazionata, del Beran, mi sembra permettere questa lettura.

Non vedo dunque perché il sigillo non debba attribuirsi a Suppiluliuma, proprio per il riferimento a Babilonia. Del resto che senso avrebbe più, sotto Mursili II, tale riferimento all'origine della tawananna, che poi era la madre?

Ritengo anzi che l'impronta frammentaria HGB I 219 appartenga allo stesso sigillo di HGB I 209 (fig. 1 i) che è di Suppiluliuma. Infatti le tracce residue della legenda geroglifica su quest'ultimo fanno parte del segno L. 16 «regina» e si trovano nella stessa identica posizione relativa rispetto al cuneiforme DINGIR.RA.

Quello che resta da interpretare è ancora la lettura dei segni costitutivi il nome geroglifico della Tawananna III di Suppiluliuma e di Mursili II, la «babilonese». Ma questo potrà chiarirsi col progresso della decifrazione del luvio geroglifico. Circa l'ipotesi di una sua identificazione con la supposta regina *Malnigal ho detto sopra.

MAŞAT 75.10 e $39{ }^{16}$ (fig. $11, \mathrm{~m}$ )

Le impronte di sigillo imperiale sulle tavolette di Maşat 75.10 e 75.39 presentano qualche problema di lettura ancora irrisolto, a causa della non perfetta impressione. La più completa delle due è

${ }^{16} \mathrm{~S}$. ALP, «Die hethitischen Tontafelentdeckungen auf dem Maşat-Höyük. Vorläufiger Bericht", Belleten XLIV (1980) 25 sgg. 
Mşt 75.39, dove l'editore dei testi ha letto chiaramente, nella metà di destra: MAGNUS REX e MONS $t u$; cioè il monogramma di un Tudbaliya, che viene identificato con il III sovrano di tal nome, il padre di Suppiluliuma. A sinistra del segno composito MAGNUS REX vi sono cinque segni geroglifici, il primo dei quali non ancora identificato con sicurezza, mentre gli altri quattro si leggono tà-túhe-pa (L. 41-325-215-334). H. Otten, apud Alp, identifico subito il nome della prima sposa ${ }^{17}$ di Suppiluliuma. Nel primo segno in alto a sinistra Alp riconosce L. 104 (la gazzella), valore fonetico sà; poiché è il primo segno delle grafie geroglifiche del teonimo Saušga, egli ipotizza una lettura Šatatuhepa, pur riconoscendo che non si conosce alcuna regina ittita di tal nome. Sull'altra impronta (Mşt 75.10) il campo centrale è ancor meno conservato, ma in compenso resta qualche segno della scritta cuneiforme della corona circolare. S. Alp legge: [N]A 4 .KIŠIB SAL.LUGAL.GA[L, avvertendo che i primi due segni non sono del tutto chiari. In effetti ho l'impressione che le tracce visibili sulla foto siano da leggere piuttosto d]u-hée-x, che integro $\left.{ }^{\mathrm{SAL}} T a-d\right] u-h e ́-p[a]$ SAL.LUGAL.GA[L. Il resto della scritta credo che dovrebbe integrarsi [ ${ }^{\mathrm{T}} T u$-ud-ha-li-ja LUGAL.GAL], ma non $\mathrm{mi}$ pare che vi sia spazio per $\mathrm{NA}_{4}$.KIŠIB. Ritornando alla legenda geroglifica, propongo di leggere il primo segno non sà, ma "DOMINA" (L. 15), anche se la sua posizione sarebbe insolita (fig. $1 \mathrm{~m}$ ). Mi sembra che questa soluzione isoli definitivamente il nome della regina Tadubepa, che una serie di indizî convergenti mostra che iniziò la sua "carriera" di tawananna prima dell'accesso al trono di Suppiluliuma. Nella copia di Alp il segno L. 15 (DOMINA) occupa la parte sinistra del campo centrale, dove mi sembra invece che si possa tutt'al più intravedere dei cunei. In quel punto si potrebbe dunque restituire la scritta cuneiforme [TI LUGAL], che farebbe da pendant a TI SAL.LUGAL, e sarebbe logica in un sigillo doppio di re e regina, come è questo.

\section{Addendum}

Mentre l'articolo era in composizione ho potuto collazionare presso il Vorderasiatisches Museum di Berlino la bulla VAT 7432,

${ }^{17}$ Si tratta piuttosto della consorte di Tudbaliya III, sopravvissuta al re, e divenuta prima tawananna di Suppiluliuma, ma non sua moglie. 
con l'impronta HGB I 159, e di questa possibilità ringrazio la direttrice Dr. Liane Jakob-Rost. La visione dell'originale mi permette di confermare l'ipotesi di lettura sopra espressa, quindi il titolo L[UG]AL.G[AL]. Ma attribuisco il $-\boldsymbol{n}$ ] $a$ di Beran alla prima parte del segno LUGAL. Lo spazio per integrare $T a-[w a-n a-a n-n a]$ diviene ora troppo esiguo. Mi sembra dunque che la vecchia lettura Tanubepat del Güterbock ridivenga probabile, ma solo per il ta iniziale e lo spazio ridotto.

Il Prof. Tahsin Özgüç mi ha d'altra parte gentilmente spedito le foto originali delle impronte Mşt 75.10 e 39, e gliene sono grato. Il risultato del rinnovato controllo è nel disegno a fig. $1 \mathrm{~m}$. Della legenda circolare è confermata senz'altro la mia lettura ${ }^{\text {SAL }}$ Ta-d]u-bép[a] SAL.LUGAL.GA[L. Quanto al primo segno geroglifico con cui sembra iniziare il nome della regina, capisco come S. Alp abbia potuto riconoscervi L. 104, ma quelle che sembrano le corna della gazzella potrebbero anche costituire delle tracce deformate di $\mathbf{L}$. 363. (MAGNUS) oppure essere delle crepe o striature dell'argilla. A sinistra di questo intravedo comunque un debole profilo del segno L. 363, ma immediatamente al di sotto non distinguo niente. Un po' più in basso, fra il bordo sinistro e i segni geroglifici he-pa, mi pare proprio di distinguere dei cunei che possono ben appartenere ai segni TI LUGAL, disposti su due piani ed orientati verso l'alto del sigillo. Non so se resti spazio sufficiente per il segno DOMINA fra il TI LUGAL e il probabile L. 363 (MAGNUS), talché affaccio l'ipotesi che per mancanza di spazio lo scriba li abbia disposti uno accanto all'altro: una soluzione "aberrante» che non ha riscontro altrove. D'altra parte se questo fosse il più antico sigillo che presenta il gruppo L. $363+15$ sarebbe forse concepibile una soluzione non ancora canonica. Il problema resta aperto, ma ritengo ad ogni modo che il segno in questione faccia parte non del nome, bensì del titolo, comunque lo si debba leggere. L'elemento decisivo è la legenda cuneiforme che, seppure incompleta, riproduce il nome ben noto di Tadubepa, mentre un supposto *Satadubepa è sconosciuto alla tradizione cuneiforme. Le implicazioni storiche del documento restano pertanto valide. 

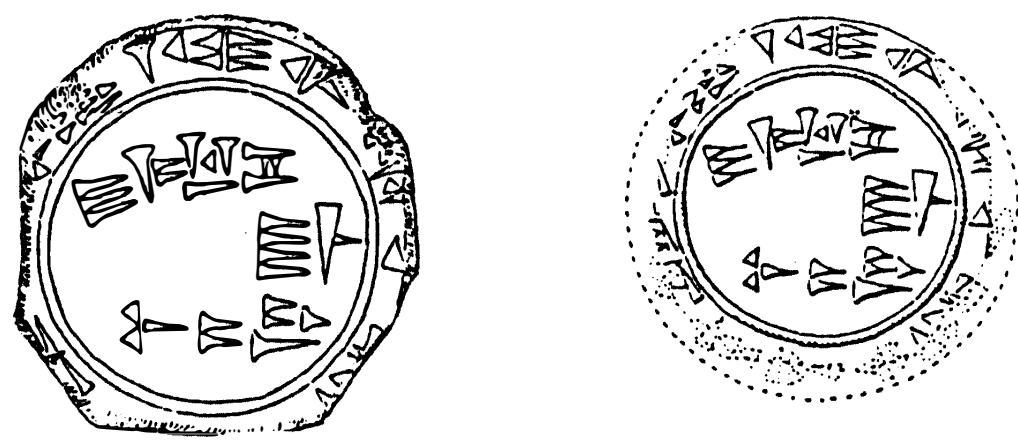

b

a

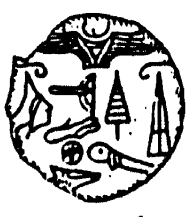

c
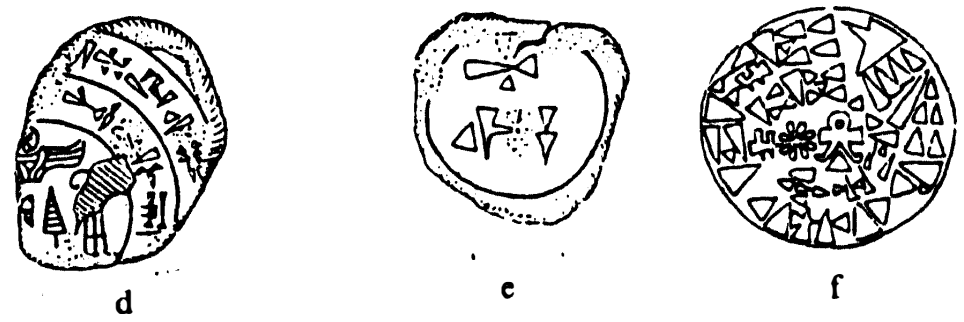

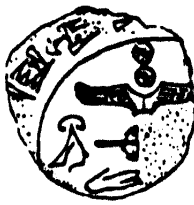

g

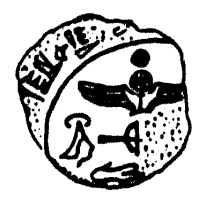

h

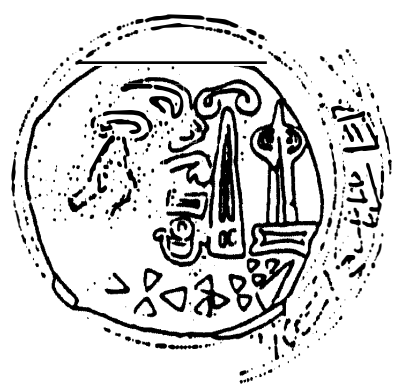

1

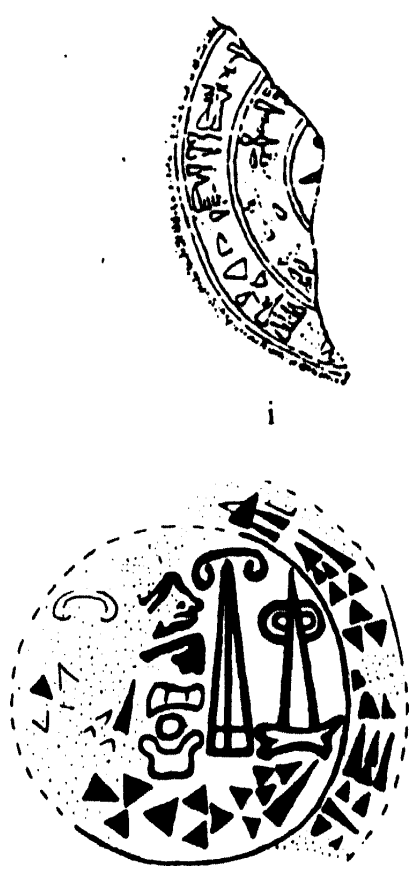

m

Fig. 1: Le illustrazioni sono tratte dalle pubblicazioni qui di seguito citate:

a) da SBo I 84; b) da HGB I 159; c) da HGB I 213a; d) da HGB I 214a; e) da SBo I 81; f) da SBo I 87 A+B+C+D; g) da SBo I 36; h) da HGB I 219; i) da HGB I 209; 1) impronta del sigillo Mşt $75.10+39$ da S. Alp, Belleten XLVI (1980) fig. 1); m) copia interpretativa provvisoria di $\mathbf{M}$. Salvini delle impronte Mşt $75.10+39$ da foto originali. 


\section{RESUMEN}

Se proponen algunas lecturas nuevas e integraciones en sellos reales hititas de Boğazköy. Probablemente no existió la reina ${ }^{*}$ Malnigal, Malni sería más bien un término de augurio del léxico hitita-luvio. La impronta del sello SBo I $36=$ HGB I 219 debe ser atribuida a Šuppiluliuma. En el sello Maşat 75.10 el nombre de la reina que acompaña al monograma de Tudhaliya III es Taduhepa y no *Šatatuhepa. Su nombre es Taduhepa en la leyenda jeroglífica y es confirmado por los trazos cuneiformes en la corona circular.

\section{SUMMARY}

Some new readings and integrations in royal Hittite seals of Boğazköy are suggested. It is likely that queen *Malnigal did not exist. Malni would rather be a term of augury of the Luvian-Hittite vocabulary. The mark of seal SBo I $36=$ HGB I 219 must be attributed to Suppiluliuma. The name of the queen which goes with Tudhaliya III monogram is not Satatuhepa but Taduhepa in seal Maşat 75.10. In the hieroglyphic inscription her name is Taduhepa and this is confirmed by the cuneiform traces in the circular crown. 